\title{
ELECTROMECHANICAL- ELECTROMAGNETIC TRANSIENT SIMULATION INTERFACE ALGORITHM OF POWER SYSTEM BASED ON PSD-PSMODEL
}

\author{
Aixia Duan1, Yongzhi Huang'2, Aiguo Du³, Yanling Duan ${ }^{4}$ \\ 1, ${ }^{4}$ North China University of Water Resources and Electric Power, Henan 450045, China; \\ ${ }^{2}$ Rundian Energy Science and Technology CO.,LTD , Henan, China; \\ ${ }^{3}$ Quzhou Power Supply Branch, State Grid Hebei Electric Power Co., Ltd. Hebei 057250, China. \\ Email: duanaixia elec22@126.com
}

\begin{abstract}
To improve the computational speed of electromechanical - electromagnetic transient simulation program of power system, based on Power System Model (PSD-PS Model) hybrid simulation program, the electromechanical - electromagnetic transient simulation interface algorithm was studied in depth. Combined with the common method of electromagnetic simulation parallel computing, according to the hybrid simulation multi-thread parallel computing method based on electromechanical transient side solution network, the multithread parallel algorithm of PSD-PS Model hybrid simulation electromagnetic transient subroutine was realized with multithread program. The results showed that when there were 7 direct current (DC) lines on the electromagnetic side, the time of serial calculation was $2740.004 \mathrm{~s}$, and the time of single-thread parallel calculation was $1175.337 \mathrm{~s}$. And the speed advantage of multi-thread parallel computing was more obvious than that of serial computing, and with the increase of DC system in the electromagnetic transient subsystem, the speed advantage was more prominent. In addition, when there were 7 DC lines in the electromagnetic transient subsystem, the time of multi-thread parallel computation was only 320.066 s. Accordingly, it can be concluded that this algorithm can greatly improve the utilization rate of processor and the speed of simulation calculation and it is very practical in engineering.
\end{abstract}

Keywords: Hybrid Simulation; Interface Algorithm; Simulation Precision PSD- PS model Advanced Digital Power System Simulator (ADPSS) Power System Analysis Software Package (PSASP).

\section{Introduction}

With the rapid development of modern power system, a large number of advanced control devices, protection devices and measurement devices, such as controllers, DC transmission controllers, relay protection devices and safety and stability monitoring equipment, including wide-area measurement devices, are widely used in the actual power grid [1]. These complex control devices improve the power grid capacity and long-distance transmission capacity, and ensure the stability, safety and quality of power supply [2]. These complex equipment and devices must be studied through simulation experiments before they are put into actual operation on the site [3]. Such experiments are usually closed-loop digital physics experiments. Therefore, the test and detection of automatic device necessarily require real-time simulation. Real - time simulation is the basis of efficient power system stability analysis and on-line dynamic security evaluation and control [4].
The research on hybrid simulation technology of power system has a history of more than 40 years. The earliest implementation of hybrid simulation technology was NETOMAC software developed by Siemens in Germany in the 1970s [5]. Heffernan first established a hybrid simulation system that considered the electromagnetic transient model of High Voltage Direct Current Transmission (HVDC), in which HVDC was modeled in detail with the method of state variables. Reeve and Adapa put forward that the interface part of the hybrid simulation should be extended outward to the alternating current (AC) system, so that the forms of the interface of the hybrid simulation can be flexible and diverse, which can prevent the waveform distortion at the interface bus from being too serious and the phase imbalance from being too large, but it increased the complexity of the interface algorithm [6]. Anderson proposed a method of frequency-dependent equivalent impedance circuit form on the electromechanical side, which can solve waveform distortion and phase imbalance at the interface in hybrid simulation with 
very little calculation [7]. At the same time, he used the method of base wave extraction based on the least square method to convert the discrete sequence data of the electromagnetic transient side into the base wave phasor required by the electromechanical side, and satisfactory results were achieved [8]. Mainstream hybrid simulation software in China includes electromechanical electromagnetic transient hybrid simulation program PS Model and Advanced Digital Power System Simulator (ADPSS) (Power System Analysis Software Package (PSASP)) independently developed by China Electric Power Research Institute (CEPRI).

PSD-PS Model hybrid simulation program is based on BPA-PSD power system transient stability analysis program and PS Model power electronics and electromagnetic transient simulation program [9]. Through hybrid simulation interface algorithm, electromagnetic transient calculation program is embedded in the electromechanical transient calculation program to realize electromechanical electromagnetic transient hybrid simulation [10]. Among them, BPA-PSD provides the system dynamic component model commonly used in electromechanical transient stability analysis, GEAR method with variable order and variable step size is adopted for numerical integration, and the differential and algebraic equations of the system are solved simultaneously. BPA-PSD technology and application has been very mature, it can complete power system simulation calculations such as single fault, complex fault and stability measures. The PS Model provides the electromagnetic transient model of all components in the DC transmission system and adopts the implicit trapezoidal algorithm with good numerical stability and mature algorithm to solve differential and partial differential equations and control system transfer function [11]. In the electromechanical - electromagnetic transient simulation program of PSD-PS Model, interface algorithm is the key technology of hybrid simulation.

\section{Electromechanical - Electromagnetic Transient Simulation Interface Based on PSD-PS Model}

\subsection{Network division of hybrid simulation of power system}

Hybrid simulation program usually divides power system network into three parts: electromechanical transient subsystem, electromagnetic transient subsystem and interface part. Under normal circumstances, the traditional AC network is put in the electromechanical transient subsystem, and the HVDC transmission system, Flexible AC Transmission Systems (FACTS) and other networks with fast response power electronic devices are put in the electromagnetic transient subsystem, and the AC bus connecting the electromechanical transient subsystem and the electromagnetic transient subsystem is taken as the hybrid simulation interface.

The PSD-PS Model hybrid simulation interface algorithm selects the position of the interface part on the AC bus on one side of the converter transformer. With such an interface location, the following advantages are mainly considered: the electromagnetic transient subsystem of the hybrid simulation mainly includes the components and control system of the HVDC transmission system, the electromagnetic transient calculation amount is relatively small, and the calculation efficiency of the hybrid simulation is high; the current and voltage on the AC bus bar on one side of the converter transformer are stable, which is beneficial to the numerical stability of electromagnetic transient simulation; the hybrid simulation interface is relatively simple. When the electromagnetic transient subsystem and electromechanical transient subsystem are equivalent to each other, the network equivalent algorithm and data conversion algorithm are relatively simple; the electromagnetic transient subsystem includes the filter at the bus bar and Switching Virtual Circuit (SVC), which can improve the analysis accuracy of the dynamic characteristics of the quick response components.

Because the location of the interface bus is selected on the AC bus on one side of the converter transformer, the waveform distortion of the converter caused by the phase imbalance and harmonics will be more serious when there is an asymmetric fault or a fault near the converter, especially when the DC system is directly connected to the weak AC system. At present, the Ultra High Voltage Direct Current (UHVDC) systems constructed and put into operation in China are directly connected with the strong AC system. The influence of phase imbalance and harmonics is small, and the resulting interface algorithm simulation error is also small. Therefore, the PSD-PS Model hybrid simulation interface algorithm selects one side AC bus of the converter transformer as the hybrid simulation interface.

\subsection{Sequential control of simulation}

The sequential control of hybrid simulation refers to the control of process sequence of electromechanical transient simulation and electromagnetic transient simulation, as well as the control of data exchange time between electromechanical transient simulation and electromagnetic transient simulation. At present, the main control methods include serial sequential 
control method and parallel sequential control method.

The computational step size of electromechanical transient simulation is usually $10 \mathrm{~ms}$, and that of electromagnetic transient simulation is usually at the microsecond level (the minimum simulation step size of PS Model is $10 \mathrm{~ms}$ ). The time consumed by electromechanical transient subroutine in hybrid simulation is much less than that of electromagnetic transient subroutine (usually within 10\%). Therefore, compared with the parallel timing sequence control method, the time consumed by simulation doesn't increase obviously. The serial timing sequence control method is adopted to ensure the high parameter accuracy of the equivalent network in the hybrid simulation and the computational accuracy of the hybrid simulation. Especially when the network topology changes, the fault timing sequence control mode can ensure that the changes of network topology be reflected in the equivalent network parameters in time, thus improving the calculation accuracy of the hybrid simulation interface algorithm.

\subsection{Calculation process of simulation interface program}

The PSD-PS Model hybrid simulation program adopts the embedded program design idea to embed the PS Model electromagnetic transient subroutine in the BPA-PSD electromechanical transient program to constitute the electromechanical - electromagnetic transient simulation program. The calculation flow chart of PSD-PS Model hybrid simulation interface program is shown in Figure 1. In the process of calculation, the program takes the calculation of one step length of electromechanical transient as the basic cycle unit. In the calculation process of electromechanical transient simulation, it also includes data preparation and processing, calculation memory allocation, calculation process and calculation result output and other operations.

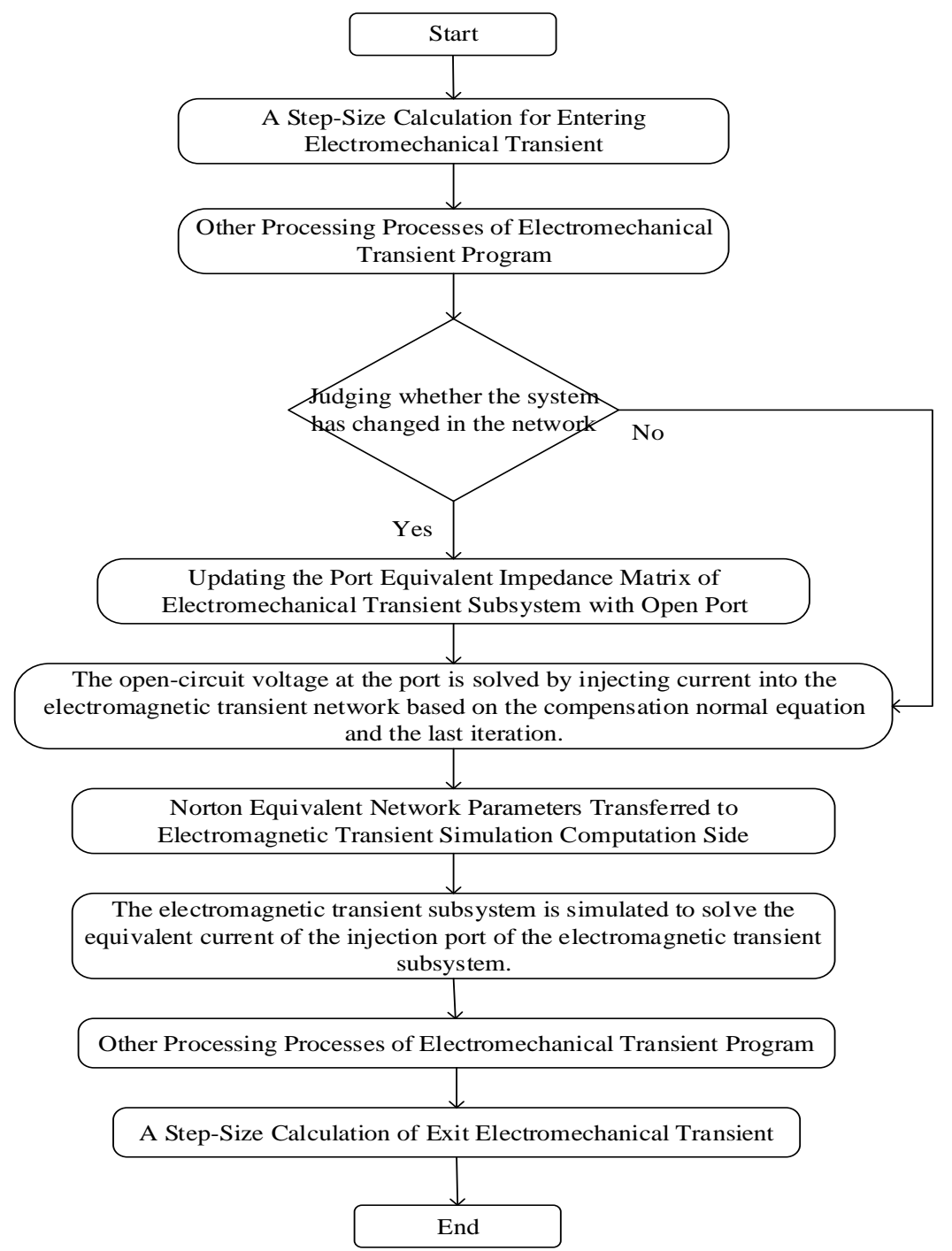

Figure 1: Computational Flow Chart of Hybrid Simulation Interface Program 


\section{In-Depth Study and Accuracy Improvement of Simulation Interface Algorithm}

\subsection{Interface algorithm for parallel implementation of linear polyphase coupling branches}

The PSD-PS Model hybrid simulation interface program converted the equivalent impedance into series resistance, inductance and capacitance. In the process of electromagnetic transient simulation, there were many problems, such as more harmonic components.
Therefore, in this Section, an interface algorithm for parallel implementation of linear polyphase coupling branches was proposed, which realized the calculation of impedance branches of multi-terminal coupling Norton equivalent circuit in the form of parallel resistance, inductance and capacitance in the electromagnetic transient subroutine.

The parallel implementation method of the following multi-terminal coupling Norton equivalent circuit impedance branches was applied to the network equivalent of the PSD-PS model hybrid simulation interface algorithm. For the equivalent circuit of linear polyphase coupling as shown in figure 2:

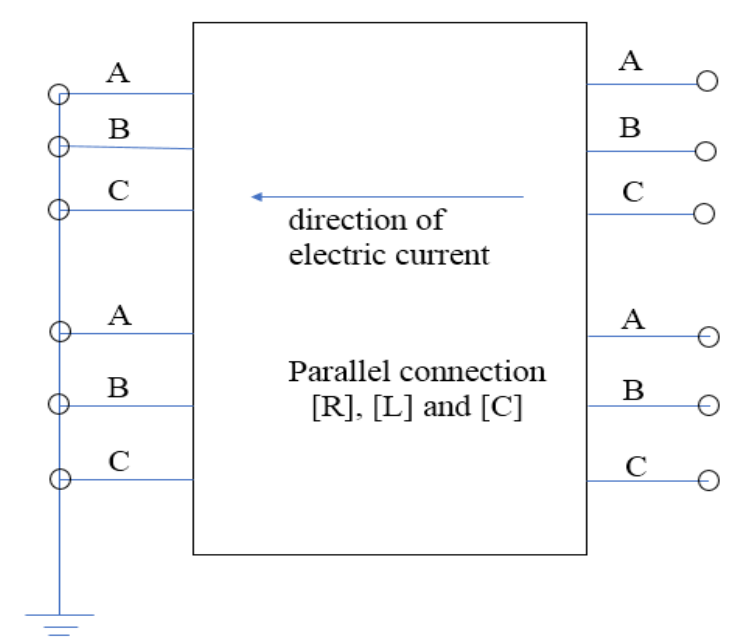

Figure 2: Linear Multiphase Coupled Equivalent Circuit

The mathematical models of resistance, inductance and capacitance in electromagnetic transient simulation are expressed by differential equations from equation (1) to equation (3):

$$
\begin{aligned}
& {\left[i_{R}\right]=[R]^{-1}\left[u_{m}\right]} \\
& {\left[u_{m}\right]=[L] \frac{d\left[i_{L}\right]}{d t}} \\
& {\left[i_{C}\right]=[C]^{-1} \frac{d\left[u_{m}\right]}{d t}}
\end{aligned}
$$

Among them, $\left[i_{R}\right],\left[i_{L}\right]$, and $\left[i_{C}\right]$ are the current vectors flowing on the resistance, inductance and capacitance respectively, and $\left[u_{m}\right]$ is the port voltage vector of resistance, inductance and capacitance.

Trapezoidal integral algorithm. The step size of electromagnetic transient simulation calculation is taken as $\Delta \mathrm{t}$. In the combined algorithm of electromagnetic transient simulation, in order to eliminate numerical oscillation, the Euler algorithm is adopted.

After discretization of differential algebraic equations (1), (2) and (3), the nodal admittance equation of the linear polyphase coupling circuit can be obtained as follows:

$$
\left([R]^{-1}+\frac{\Delta t(1+\alpha)}{2}[L]^{-1}+\frac{2}{\Delta t(1+\alpha)}[C]\right)\left[u_{m}\right]+\left[h_{L}(t)\right]+\left[h_{C}(t)\right]=0
$$

By deducing the conclusion from the above formula, the parallel circuit of polyphase coupling inductance, capacitance and resistance in figure 2 can be converted into the difference equation used in electromagnetic transient simulation calculation, as shown in equation (4).

After adopting the algorithm of parallel implementation of the linear polyphase coupling branches, the PSD-PS Model hybrid simulation 
interface program can deal with the asymmetry and mutual coupling of the equivalent circuits when the electromechanical side subsystem was equivalent to the electromagnetic side. Compared with the serial circuit implementation method, the interface algorithm with parallel implementation of linear polyphase coupling branches was adopted. The harmonic content in the electromagnetic transient simulation results was greatly reduced and the harmonic stability was obviously enhanced. At the same time, in order to facilitate the further study of this related problem, the PSD-PS Model hybrid simulation interface program kept two modules, that is, parallel implementation algorithm and serial implementation algorithm, for self-selection.

\subsection{Negative resistance processing algorithm based on current correction for port equivalent admittance matrix}

Generally, when the electromagnetic transient simulation subroutine of PSD-PS Model was diverging due to negative resistance, the negative resistance was set to zero. In this method, the equivalent admittance matrix of ports was modified approximately, so there would be some simulation errors. In this section, a negative resistance processing method of port equivalent impedance based on current correction was proposed, which can deal with the negative resistance phenomenon in port equivalent admittance matrix by modifying the current source in Norton equivalent circuit, so that the PSD-PS Model hybrid simulation interface algorithm can ensure the calculation accuracy in the process of equivalent calculation.

Suppose that at a certain moment, the equation of the electromechanical transient subsystem after the port equivalent and the electromagnetic transient subsystem at the port can be expressed as equation (5):

$$
[Y][V]=\left[I_{N}\right]+\left[I_{E}\right]
$$

Among them, $[\mathrm{Y}]$ is the port equivalent admittance matrix of the electromechanical transient subsystem at the interface, $\left[\mathrm{I}_{\mathrm{N}}\right]$ is the current vector in the Norton equivalent circuit of the electromechanical transient subsystem, $\left[\mathrm{IE}_{\mathrm{E}}\right]$ is the current vector injected into the port of the electromechanical transient subsystem calculated by electromagnetic transient simulation, and $[\mathrm{V}]$ is the port voltage vector at the interface calculated by the electromechanical transient subsystem.

If it was found that the admittance matrix [Y] after a series of transformations led to the occurrence of negative resistance in the electromagnetic transient calculation process, the admittance matrix $[Y]$ was modified to be the equivalent admittance matrix ' $[\mathrm{Y}]$ after the elimination of negative resistance. After the matrix was modified, corresponding changes were made to equation (5).

The modified equation was as follows:

$$
\left[Y^{\prime}\right][V]+\left([Y]-\left[Y^{\prime}\right]\right)[V]=\left[I_{N}\right]+\left[I_{E}\right]
$$

Further modification was made to equation 6 , as shown in equation 7 below:

$$
\left\{\begin{array}{c}
{\left[Y^{\prime}\right][V]=\left[I_{N}^{\prime}\right]+\left[I_{E}\right]} \\
{\left[I_{N}^{\prime}\right]=\left[I_{N}\right]-\left([Y]-\left[Y^{\prime}\right]\right)[V]}
\end{array}\right.
$$

After the modification of the equivalent current phasor above, the solution of equation (6) in iterative calculation was exactly the same as that of equation (7) in iterative calculation. This method can not only ensure the equivalent calculation accuracy of the PSD-PS Model hybrid simulation interface algorithm, but also solve the electromagnetic transient result divergence or program suspension caused by the negative resistance generated in the equivalent admittance matrix in the electromagnetic transient subsystem.

This method can deal with the slight negative resistance problem occurring in the equivalent admittance matrix. If a large negative resistance appeared in the admittance matrix of the equivalent port, this method was not applicable. At this time, it was necessary to find a large negative load near the hybrid simulation interface location and convert the negative load into the actual generator equipment model.

\subsection{Verification method for correctness of hybrid simulation interface algorithm}

Electromagnetic transient simulation and electromechanical transient simulation belonged to two different types of computing methods. There were great differences between them in simulation step size and simulation model (generator, circuit and DC system model, etc.). In addition, some key techniques in electromagnetic transient simulation were still in the closed state.

The research on electromagnetic transient model and simulation algorithm of electromagnetic transient part of electromagnetic transient program or hybrid simulation program independently developed in China was still in the stage of continuous exploration and improvement.

If the hybrid simulation results of the HVDC transmission system were directly compared with the electromagnetic transient simulation results, there must be some inconsistencies. 
The errors between hybrid simulation and pure electromechanical transient simulation mainly included two parts. One were the calculation errors caused by different simulation algorithms in electromechanical transient simulation and electromagnetic transient simulation of the same $\pi$ AC line model; the other were the errors caused by hybrid simulation interface algorithm, including errors caused by network equivalence and errors caused by data interaction conversion. If the error in the first part was $\sigma_{1}$ and the error in the second part was $\sigma_{2}$, then the calculation error $\sigma_{0}$ caused by the hybrid simulation interface algorithm was related to the equation (8).

$$
\left|\sigma_{1}-\sigma_{2}\right| \leq \sigma_{o} \leq\left|\sigma_{1}+\sigma_{2}\right|
$$

Therefore, the maximum error value of each parameter can be obtained by counting different types of faults, different fault locations (near and far of the interface), and different electromagnetic transient subsystems (number and location of AC transmission lines in the electromagnetic transient subsystem were different). The maximum error between the hybrid simulation and the pure electromechanical transient simulation results can be used as the upper limit of the maximum error of the electromechanical - electromagnetic transient hybrid simulation program interface algorithm, so as to verify the accuracy of the interface algorithm.

\subsection{Verification of computational correctness of hybrid simulation interface algorithm}

The IEEE9 was used for example test. For hybrid simulation calculations, the AC line: Bus 2 - Bus C were placed in the electromagnetic transient subsystem and the rest was placed in the electromechanical transient subsystem.

The fault was set at the bus A, and A-phase ground fault occurred at $1.0 \mathrm{~s}$, the fault was eliminated at $1.1 \mathrm{~s}$, and the simulation time was $6.0 \mathrm{~s}$.

The pure electromechanical transient simulation calculation, the hybrid simulation calculation before improving the precision and the hybrid simulation calculation after improving the precision were carried out respectively.

Among the three simulation results, the positive sequence voltage of bus 2 , the power angle of generator 2 and the active power of bus 1 - bus $\mathrm{A}$ were compared, and the curves of the parameters were as follows:

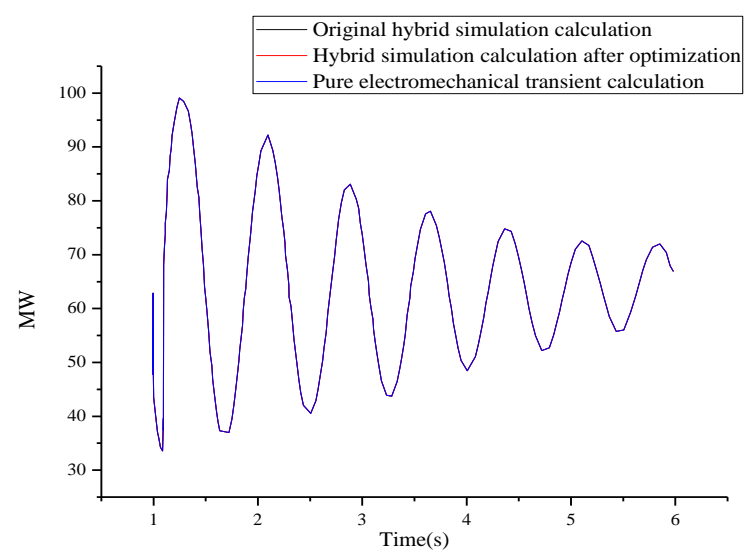

Figure 3: Active power curve of bus bar

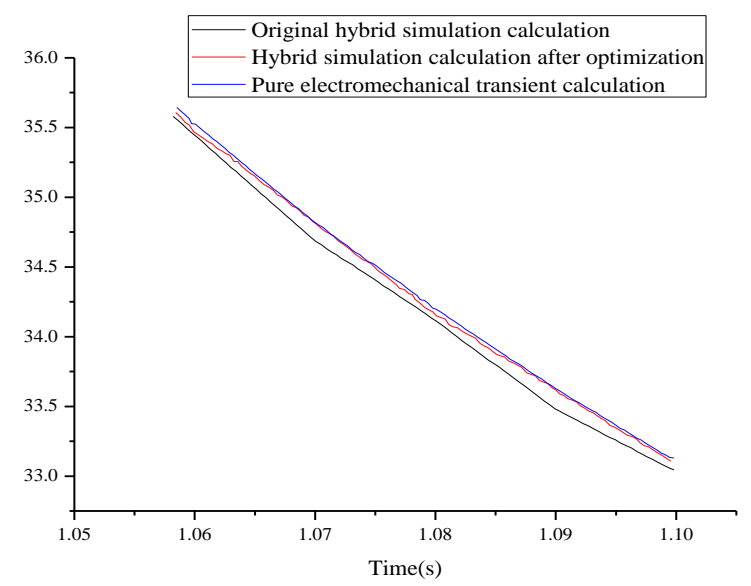

Figure 4: Near Breakdown Enlarged drawing about Contrast Diagram of Active Power Curve of Bus

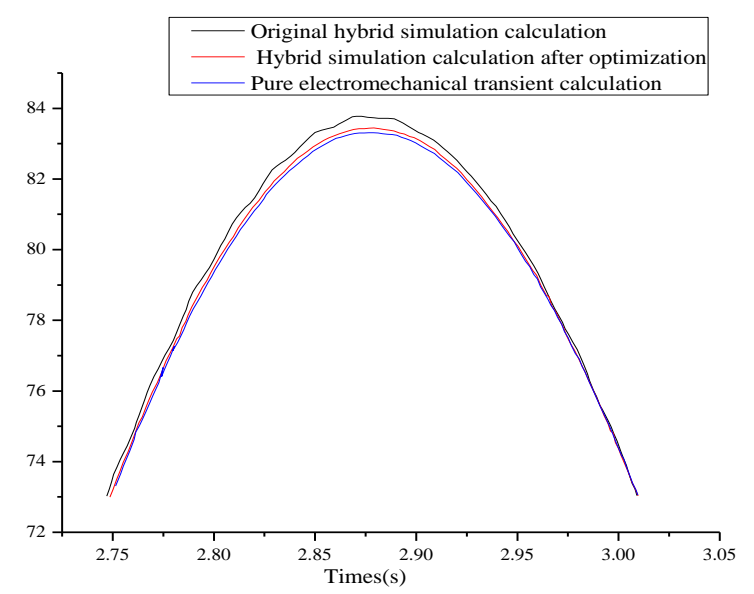

Figure 5: Near 2.8s Enlarged drawing about Contrast Diagram of Active Power Curve of Bus

The comparison in figure 5 showed that the curves of the original hybrid simulation results and the improved hybrid simulation results basically coincide with the curves of the pure electromechanical transient simulation results. After the statistics of the error between the simulation 
results, the maximum error between the original hybrid simulation results and the improved hybrid simulation results and the pure electromechanical transient hybrid simulation results was within $1 \%$. So, in the process of hybrid simulation calculation, the error of hybrid simulation caused by interface algorithm was also within $1 \%$, which can fully meet the requirements of power system for simulation tools. Compared with the original hybrid simulation results, the hybrid simulation curve with improved precision overlapped better with the pure electromechanical transient simulation curve, and the error generated by the interface algorithm was smaller. Especially in one or two cycles after the network topology changed, there was a large jitter in the original hybrid simulation results, and the simulation error reached the maximum value at this time. However, the improved hybrid simulation eliminated the larger jitter in the original hybrid simulation within one or two cycles after the network topology changed. The simulation results showed that the computational accuracy of the improved PSD-PSModel hybrid simulation interface program was significantly improved, which met the requirements of large-scale power grid application.

\section{Hybrid Simulation Multi-Thread Parallel Computing Technology}

In order to make full use of the processor's multicore processing technology and improve the simulation speed of electromagnetic transient subroutine in hybrid simulation, it is possible to simulate large-scale AC/DC hybrid simulation on PC.

In this section, parallel computing methods commonly used in electromagnetic transient simulation were studied. According to the characteristics of PSD-PS Model hybrid simulation, a parallel computing method in the electromechanical transient side was proposed to realize the mutual decoupling between different DC systems. And the method of multithreads computing was adopted to carry on multithread programming to the hybrid simulation interface program.

From the two aspects of simulation accuracy and simulation speed, a large power grid example test was conducted for the PSD-PS Model hybrid simulation program of multi-thread parallel computing.

\subsection{Common parallel method for electromagnetic transient simulation}

In the electromagnetic transient simulation of large-scale power grid, each partition has relatively independent network characteristics. Therefore, the large-scale power system is divided into several small systems, parallel computing is carried out on different computing cores, and then the simulation results between each small system are exchanged through the contact module. After such parallel processing, the speed of electromagnetic transient simulation calculation can be significantly improved.

At present, three parallel computing methods for network segmentation are mainly proposed at home and abroad: long transmission line decoupling parallel algorithm, MATE (Multi-Area Theremins Equivalent) parallel algorithm, and node splitting parallel algorithm.

\subsection{Hybrid simulation multi-thread parallel algorithm for electromechanical transient side solution network}

The parallel calculation of the electromagnetic transient subroutine can segment the electromagnetic side subsystem and perform parallel calculation on different divided regions.

When the hybrid simulation program performed electromagnetic transient simulation on the DC system, the following characteristics existed between the DC systems:

The converter stations of multiple DC systems were not on the same AC node.

In the hybrid simulation, the two ends of the DC system were located at different ports; the DC system run independently, and there was no mutual coordination between the control and protection systems, and complete decoupling can be realized in the electromagnetic transient simulation calculation; the electrical connection between multiple DC lines was realized through the AC network in the electromechanical side subsystem.

In this section, a hybrid simulation multi-thread parallel algorithm of electromechanical transient side solution network was proposed based on the above characteristics, as shown in figure 6. On the electromechanical side, the DC system in the electromagnetic transient subsystem was divided to achieve mutual decoupling between the DC systems.

Simulation calculation was carried out in different electromagnetic transient subroutines, which not only reduced the calculation scale of electromagnetic transient simulation, but also formed parallel calculation among different DC systems, and improved the simulation speed of electromagnetic transient subroutine. 
At the same time, taking advantage of the multithreaded computing of multi-core processors, multithreaded parallel computing was used to further accelerate the simulation speed of the electromagnetic transient subsystem.

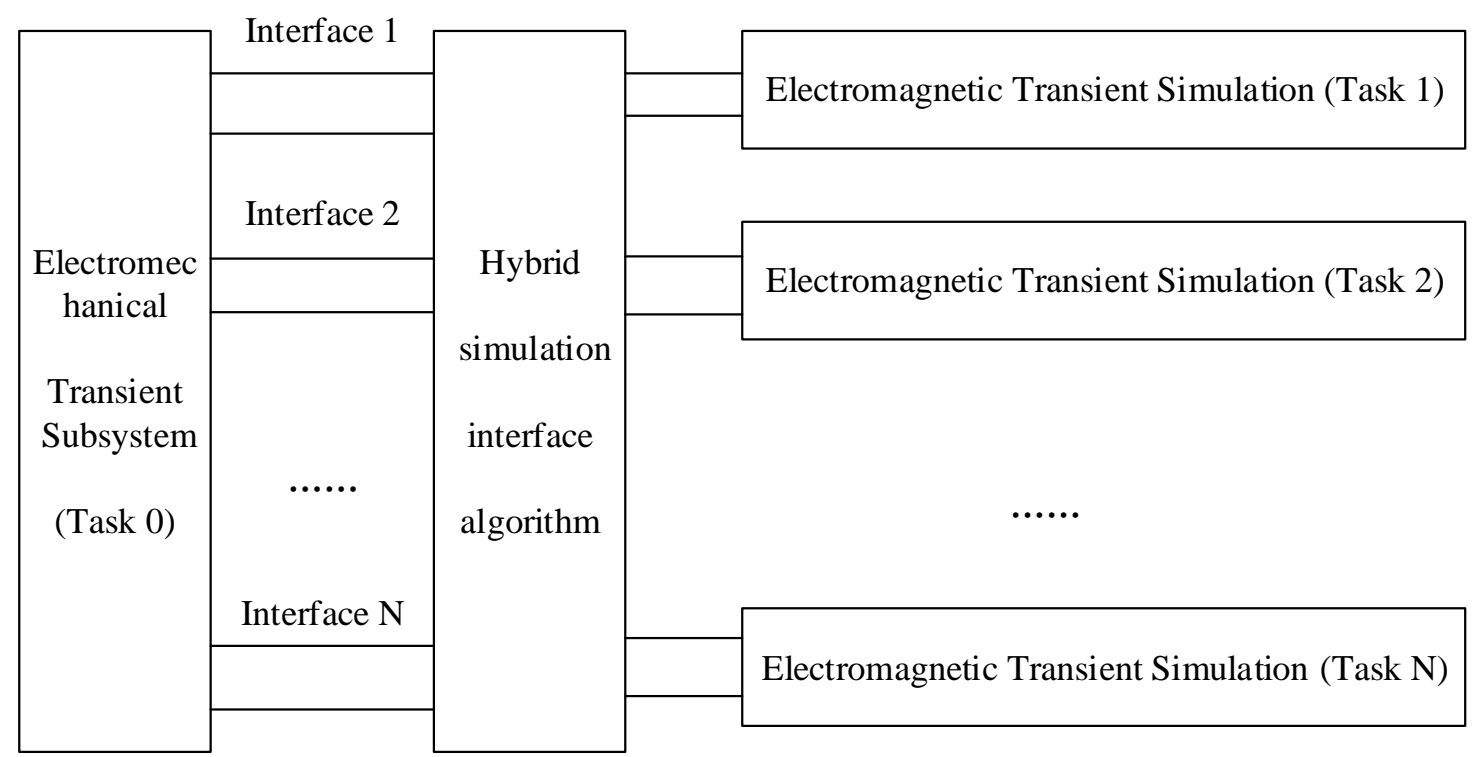

Figure 6: Hybrid simulation multi-threaded parallel algorithm for electromechanical transient side unwinding

According to the above analysis, the proposed multi-threaded parallel algorithm mainly includes six key technologies, that is, segmentation method of the electromagnetic side subsystem, multi-task division method for parallel computing, parallel computing thread control mechanism, parallel computing of electromechanical transient subsystem to the electromagnetic lateral equivalence algorithm, and parallel computation of electromagnetic transient subsystem to electromechanical side equivalence algorithm.

By applying the above techniques to the interface program, the multithreaded parallel computation of the hybrid simulation program can be realized.

In the parallel segmentation method of the electromagnetic side subsystem, when the electromagnetic transient subroutine was serially calculated, the electromagnetic side subsystem was equivalent to a multi-port network to the electromechanical side.

The electromagnetic models of all HVDC transmission systems in the network were solved in the same differential equations.

In the electromagnetic transient subsystem, the number of DC systems was large, and in the differential and partial differential equations formed, the electrical nodes and the control transfer functions between the DC systems were coupled together, and the equations were large in scale. Parallel segmentation method was used to segment the electromagnetic transient subsystem from the electromechanical side.

The segmentation point was set at the AC interface bus on the electromechanical side, and each pair of ports corresponded to a segmentation area.

After segmentation, the electromechanical side of the electromagnetic side subsystem is equivalent to multiple two-terminal networks, so as to achieve complete independence between different DC systems in the electromagnetic transient subsystem.

When the program was implemented, the electromagnetic transient subsystem was used for segmentation, as shown in Figure 7.

The program separately stored the data and control files of different electromagnetic segmentation systems and opened up different memory spaces for each group of electromagnetic segmentation systems.

Each group of electromagnetic segmentation system can independently call the electromagnetic transient subroutine for simulation calculation and realize the electromagnetic transient parallel calculation function of multiple DC systems from the program. 


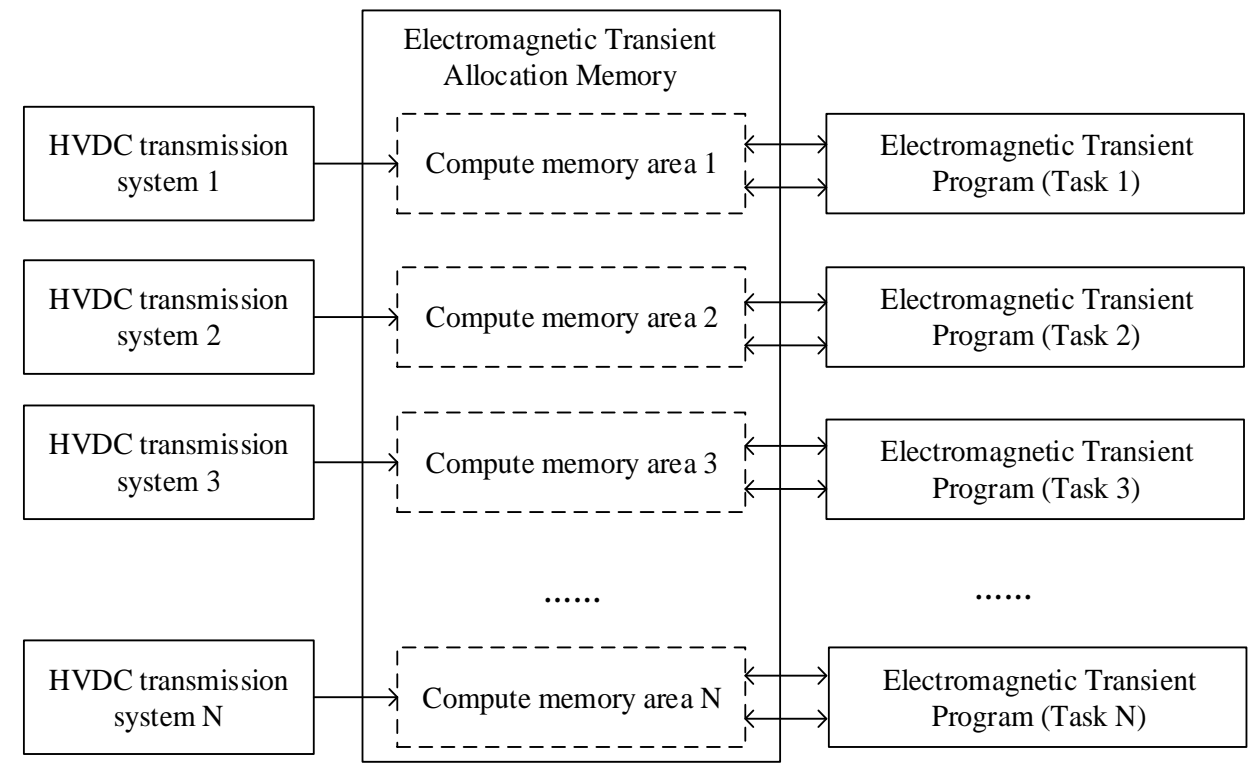

Figure 7: Programming Implementation of Parallel Segmentation

In order to ensure the computational accuracy of the hybrid simulation interface algorithm, the hybrid simulation interface algorithm of multi-thread parallel computing still adopted the serial calculation method in the overall sequential control of electromechanical transient calculation and electromagnetic transient calculation. It included normal mode and fault mode. When the electromagnetic transient subroutine was calculated, it was necessary to modify the flow control of the electromagnetic transient subroutine according to the parallel calculation method. And the process control method of parallel computation of multiple electromagnetic transient programs should be adopted.

As for the thread control mechanism of multithreaded parallel computing, the PSD-PS Model hybrid simulation parallel computing method adopted the kernel level thread implementation model in multi-threaded computing. The thread switch of kernel level thread mechanism was controlled by the kernel processor of CPU. When the thread was switched, the processor changed from the user state to the kernel state. After the thread was switched, the processor returned to the user state from the kernel state. In the kernel-level thread control mechanism, when a thread in a core was computationally blocked, it didn't affect the operation of other threads at all. Compared with the user-level thread control mechanism, the kernellevel thread control mechanism had the advantages of simple multi-thread programming, high utilization of multi-core CPU, and small influence of parallel threads on each other.

The hybrid simulation interface program divided the hybrid simulation computing task into multiple tasks. After being allocated to multiple computing threads, when a thread needed to be evaluated, it was only necessary to start the thread, transfer the task of the thread to the kernel space, and the computer kernel would automatically allocate the processor's thread for the calculation. When this thread control mechanism was adopted, the controller used different threads of the processor in consideration of factors such as the temperature and frequency of the processor. In particular, when the total number of CPU threads was more than the number of user task threads, the controller would use all the threads of CPU interactively, thereby avoiding the problem that the CPU was overheated due to the high-load operation of a certain thread.

\subsection{Test of simulation accuracy and calculation speed of hybrid simulation parallel algorithm}

In this section, a large AC-DC parallel operation power grid composed of north China power grid, central China power grid and east China power grid was adopted as a simulation example of parallel computing test. The grid structure of this example was complex and large-scale, including multiple UHVDC transmission systems. Through the simulation test of this example, the performance of PSD-PS Model hybrid simulation multi-thread parallel computing technology in the actual power grid application can be obtained.

In the example, seven UHVDC systems of Linfeng, Sanchang, Yihua, Genan, Fufeng, Jinsu and Binjin were concentrated. In the hybrid simulation, the seven DC systems were placed in the electromagnetic transient subsystem for electromagnetic transient simulation, and the remaining power grids were placed in the electromechanical transient subsystem for 
electromechanical transient simulation. This example was used to test the correctness and speed advantage of the multi-thread parallel computing algorithm of the electromechanical side solution network.

The fault location of the example was set in the east China power grid where the drop points of DC system were concentrated. Specifically, the fault was set at $525 \mathrm{kV}$ of the AC bus, and a three-phase shortcircuit fault occurred at 2.0 s with a duration of $0.1 \mathrm{~s}$. In the PSD-PS Model hybrid simulation calculation, the initialization time of the electromagnetic transient simulation was $2.0 \mathrm{~s}$, and the simulation duration of the hybrid simulation was $6.0 \mathrm{~s}$.

The main test contents were as follows: firstly, the simulation correctness verification of hybrid simulation parallel algorithm. In the case of the same DC system in the electromagnetic transient subsystem, the hybrid simulation results of the multi-thread parallel computing and the hybrid simulation results of the original serial computing method were compared to obtain the simulation correctness of the hybrid simulation parallel algorithm; secondly, speed advantage test of hybrid simulation multi-thread parallel algorithm. The simulation time consumed by the hybrid simulation program of original serial computing method, singlethread parallel computing method and multi-thread parallel computing method with the increase of the number of DC systems in the electromagnetic transient subsystem was compared, so as to obtain the speed advantage of hybrid simulation with multithread parallel algorithm.

Firstly, the correctness of the hybrid simulation multithreaded parallel algorithm was verified.

Seven DC systems (Linfeng, Sanchang, Yihua, Genan, Fufeng, Jinsu and Binjin) were set in the electromagnetic transient subsystem, respectively, for hybrid simulation calculation of the original serial calculation method and hybrid simulation calculation of multi-thread parallel calculation method.

In the comparison of simulation results, the parameters of DC voltage, DC current, DC power, $\gamma$ angle (extinction angle on the inverter side) and $\alpha$ angle (firing angle on the rectifier side) in the electromagnetic transient subsystem were compared, and the simulation differences of the two hybrid simulation in the electromagnetic transient subsystem were obtained. By comparing the parameters of node voltage, line power and swing angle of generator in the electromechanical transient subsystem, the simulation differences of the two hybrid simulations in the electromechanical transient subsystem were obtained.

By comparing the parameter curves of the simulation results, it was shown that the hybrid simulation result parameter curves of the multithread parallel computing algorithm basically coincided with the hybrid simulation result parameter curves of the original serial computing algorithm, and the error was very small (The maximum error is within 1\%). Several typical parameter curves were selected shown in Figure 810.

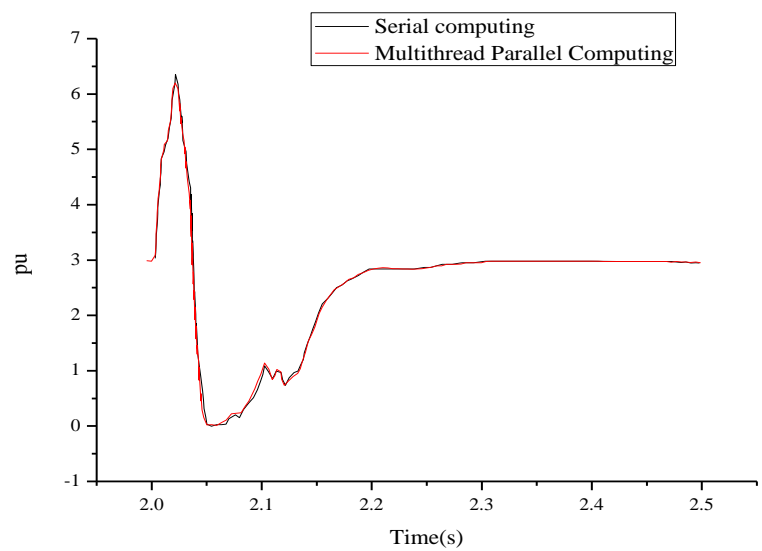

Figure 8: Comparison of DC Current Curves on Inverter Side of Three-way DC System

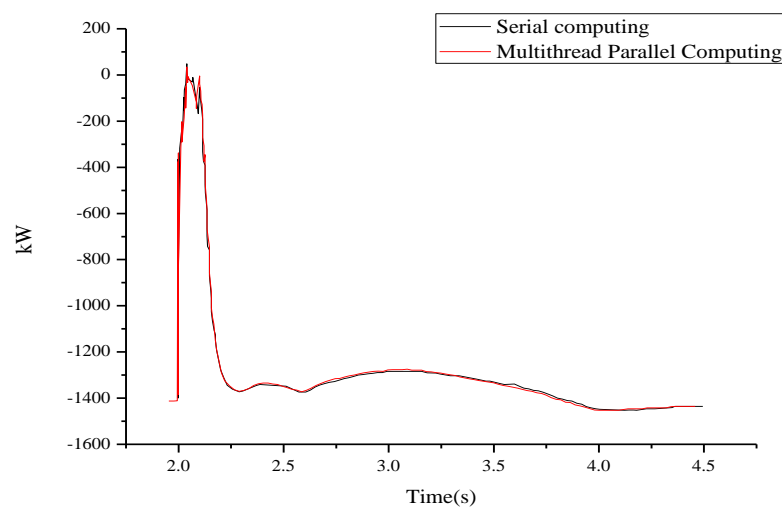

Figure 9: Comparison of DC Power Curves on Inverter Side of Three-way DC System

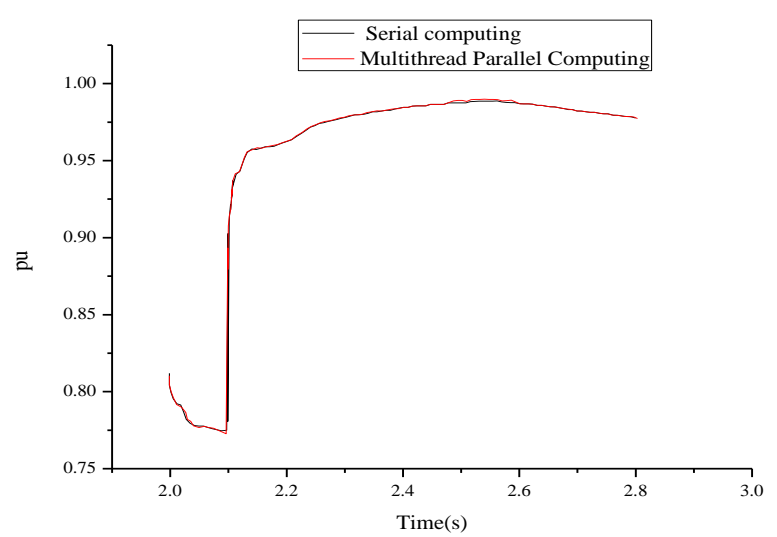

Figure 10: Curve Comparison of Node Su Taixing's Positive Sequence Voltage

In the curve comparison results of Figure 8-10, there were slight differences between the results curves of the two hybrid simulation methods, which 
were mainly caused by the parallel computing algorithm. When the electromagnetic transient subsystem in the serial computing algorithm was simulated, there were small impedance that were coupled with each other among the multiple DC systems, and the control systems of multiple DC systems interacted with each other. After adopting the parallel computing algorithm of electromechanical side disconnection network, complete decoupling was achieved between DC systems, which made the electrical parts and control systems of multiple DC systems completely independent in the electromagnetic transient simulation process and reduced the mutual interference in the calculation process.

The error between the hybrid simulation with parallel computing and the original serial hybrid simulation was very small, and the maximum error was within $1 \%$. After adopting the multi-thread parallel computing algorithm of electromechanical transient side solution network, the hybrid simulation results were basically consistent with the simulation results of the original serial computing method, which verified that the multi-thread parallel computing method proposed in this section can ensure the correctness of the simulation results in the hybrid simulation. Therefore, the hybrid simulation multi-thread parallel algorithm separated the multiple DC systems without direct connection on the electromechanical side, and the method of implementing multiple parallel DC calculations was correct in terms of simulation results.

Then, the speed advantage of the simulation multithreaded parallel algorithm was tested.

The original serial computing method used only a single thread of the processor in the hybrid simulation. Multi-thread parallel algorithm adopted parallel algorithm and multi-thread processing technology to improve the simulation speed. In hybrid simulation, multiple threads of the processor can be used for calculation at the same time to improve the utilization rate of the processor and the simulation speed.

In this section, the hybrid simulation computing speed of the three methods was tested: original serial computing method hybrid simulation, singlethread parallel computing method hybrid simulation and multi-thread parallel computing method hybrid simulation. For each hybrid simulation, the time consumed by hybrid simulation calculation was tested when the number of DC systems in the electromagnetic transient subsystem was different. Therefore, the speed advantage of hybrid simulation with parallel computing method and multi-thread processing technology was obtained from different angles.

The test computing environment was a multicore server, configuration: two Intel Xeon CPUs, 12 physics cores, 24 calculation threads, 4G DDR3 memory, and 64-bit Windows 7 enterprise edition system.

After the speed test of the hybrid simulation, the consumption time of the three forms of hybrid simulation (the serial calculation method, the singlethread parallel calculation method and the multithread parallel calculation method) were counted when the number of DC systems in the electromagnetic transient subsystem was different. The statistical results were as follows: the number of DC systems in the electromagnetic transient subsystem increased from 1 to 7 . And the comparison results of simulation time in different hybrid simulation forms were as follows.

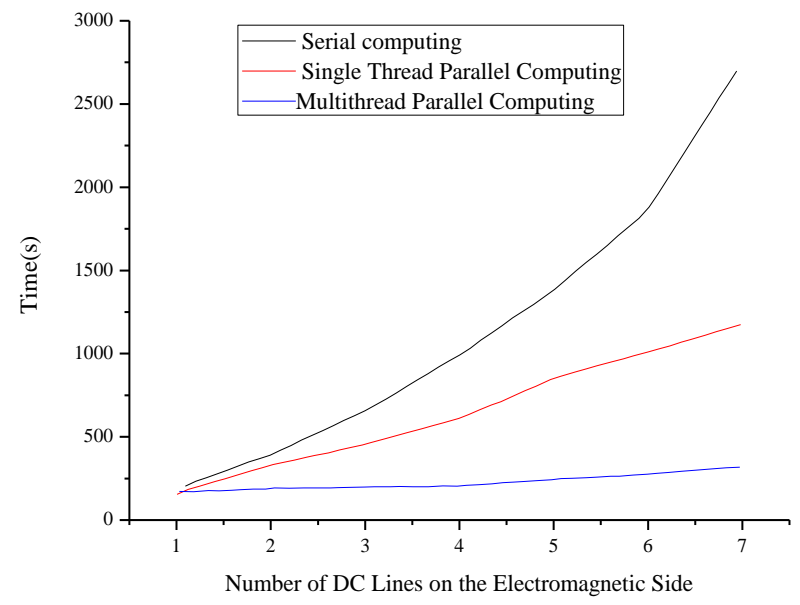

Figure 11: Comparison Curve of Hybrid Simulation Speed Test

Figure 11 showed that the consumption time of hybrid simulation of serial calculation method increased exponentially with the increase of the number of DC systems on the electromagnetic side. With the increase of the number of DC systems on the electromagnetic transient side, the consumption time of hybrid simulation of single-thread parallel computing presented a trend of nearly linear increase. And with the increase of the number of electromagnetic transient side DC systems, the hybrid simulation time of multi-thread parallel computing showed a trend of almost no increase.

According to the analysis of test results, it was assumed that the computational consumption time of electromechanical transient was $M$, the electromagnetic transient calculation time of a DC system was $\mathrm{N}$, and the calculation consumption time of system equivalence and data exchange was $\mathrm{P}$. Where $\mathrm{P}$ was far less than $\mathrm{N}$, and the number of DC systems in the electromagnetic transient subsystem was $\mathrm{x}$.

In the hybrid simulation of serial calculation method, there was a coupling calculation relation between all the DC control systems, and the calculation amount of the control system increased with the number of DCs in a square relationship. 
Therefore, the hybrid simulation time of the serial computing method can be approximated by formula (8):

$$
T_{s s}=M+[(N+P) x]^{2}
$$

In the hybrid simulation of single-thread parallel computing method, the DC system was decoupled due to the parallel computing method. Therefore, with the increase of the number of DC systems, the electromagnetic transient consumption time increased linearly. Since the electromagnetic transient calculations were all in one thread and were in a serial calculation relationship with the electromechanical transients, the hybrid simulation time of the single-thread parallel computing method can be approximated by equation (9):

$$
T_{d s}=M+[(N+P) x]
$$

In the hybrid simulation of multi-thread parallel computing method, multi-thread processing technology was added on the basis of single-thread parallel computing. When the number of CPU threads was sufficient, the electromagnetic transient consumption time didn't increase with the number of DC systems. Therefore, the hybrid consumption time of the multi-threaded parallel computing method can be approximated by formula (10):

$$
T_{d s}=M+N+P x
$$

After the above theoretical analysis on the consumption time of hybrid simulation calculation, three approximate formulas for the consumption time of hybrid simulation calculation were obtained. The increasing trend described in equations (8) to (10) was basically consistent with the increasing trend of three hybrid simulation consumption times obtained from simulation tests. The theoretical analysis results and the actual test results were mutually verified. It was concluded that with the increase of the DC system in the electromagnetic transient subsystem, the serial computing hybrid simulation consumption time increased approximately squarely. The consumption time of hybrid simulation of single thread parallel computing increased approximately linearly, while that of hybrid simulation of multiple threads parallel computing didn't increase approximately.

By adopting the parallel algorithm method, the mutual coupling calculation between the DC systems in the electromagnetic transient subsystem was eliminated, and the electromagnetic transient computation was reduced. Therefore, single-thread parallel computing was much faster than serial computing, and with the increase of the number of DC on the electromagnetic side, the advantage was more obvious. When there were 7 DC lines on the electromagnetic side, the serial computing time was $2740.004 \mathrm{~s}$, and the single thread parallel computing time was 1175.337 s. After adopting multi-thread parallel computing technology, different DC systems were allocated to different computing threads for parallel computing. Therefore, with the increase of DC system in the electromagnetic subsystem, the consumption time of hybrid simulation increased very little. The results showed that the speed advantage of multi-thread parallel computing was more obvious than that of serial computing, and with the increase of DC system in the electromagnetic transient subsystem, the speed advantage of multithread parallel computing was more prominent. When there were 7 DC lines in the electromagnetic transient subsystem, the time of multi-thread parallel computation was only 320.066 s, which was nearly 9 times shorter than the serial computation and had a strong practical value.

\section{Conclusion}

China's UHV AC-DC parallel operation grid is developing rapidly and the number and capacity of HVDC projects are increasing. In this study, based on the PSD-PS Model hybrid simulation program developed by the electric power research institute, the computational accuracy and computational speed of hybrid simulation interface algorithm were deeply studied. The PSD-PS Model hybrid simulation program was greatly improved from the two aspects of computational accuracy and computational speed of hybrid simulation interface algorithm, which made it had stronger engineering practical value.

The main research was as follows: an interface algorithm of parallel implementation of linear polyphase coupling branches was proposed to realize the electromagnetic transient model of parallel impedance in Norton equivalent network when the electromechanical side subsystem was equivalent to the electromagnetic side, so as to reduce the oscillation and harmonic content in electromagnetic transient simulation; a negative resistance processing method based on current correction was found for a port equivalent admittance matrix. The negative resistance equivalent processing in the equivalent admittance matrix improved the computational stability of hybrid simulation; a hybrid simulation interface algorithm based on pure electromechanical transient simulation was proposed; the test results of the improved PSD-PSModel hybrid simulation interface algorithm showed that the improved hybrid simulation interface algorithm had very high computational accuracy; the hybrid simulation parallel algorithm of electromechanical transient side solution network was used to decouple the electromagnetic transient subsystem in PSDPSModel hybrid simulation to realize parallel calculation of different DC systems, reduce the calculation amount of electromagnetic transient simulation, and improve the calculation speed of hybrid simulation. Multithread parallel computation 
programming was carried out on the PSD-PSModel hybrid simulation program to complete the thread allocation and control of multithread parallel computation in hybrid simulation and improve the utilization rate of processor and the computation speed of hybrid simulation.

In this research, through the in-depth study of interface algorithm, the calculation accuracy and calculation stability of the interface algorithm were improved. The multi-thread parallel computing method greatly improved the calculation speed of hybrid simulation and had high engineering value. At present, electromechanical - electromagnetic transient hybrid simulation technology is still in the stage of continuous research and improvement.

\section{Acknowledgement}

Research startup subject of Yangtze Normal University: 2017KYQD16

\section{References}

[1] Zhuoyuan, G. U., Yong, T., \& Wenzhuo, L. (2015). Electromechanical Transient-Electromagnetic Transient Hybrid Simulation of Doubly-Fed Induction Generator. Power System Technology, 77(11), 1134-1141.

[2] Sun, X. Y., Zhu, Y. Q. (2014). Research on General Interface Model Suitable for Electromechanical Transient Simulation. Applied Mechanics and Materials, 551, 5.

[3] Ji, L., Niu, D. X., \& Xu, M. (2015). An optimization model for regional micro-grid system management based on hybrid inexact stochasticfuzzy chance-constrained programming. International Journal of Electrical Power \& Energy Systems, 64(64), 1025-1039.

[4] Tadayon, N., Khoshroo, S., \& Askari, E. (2013). Power management in SMAC-based energyharvesting wireless sensor networks using queuing analysis. Journal of Network \& Computer Applications, 36(3), 1008-1017.

[5] Boonstra, P. S., Taylor, J. M., \& Mukherjee, B. (2013). Incorporating auxiliary information for improved prediction in high-dimensional datasets: an ensemble of shrinkage approaches. Biostatistics, 14(2), 259-272.

[6] Sangid, M. D., Maier, H. J., \& Sehitoglu, H. (2011). A physically based fatigue model for prediction of crack initiation from persistent slip bands in polycrystals. Acta Materialia, 59(1), 328-341.

[7] D, Alessandro. A., Luzio, D., \& D, Anna. G. (2011). Seismic Network Evaluation through Simulation: An Application to the Italian National Seismic Network. Bulletin of the Seismological Society of America, 101(3), 1213-1232.

[8] Lin, J., Sun, Y., \& P, Sorensen. (2011). Simulation of power fluctuation of wind farms based on frequency domain. Part one. Model and analysis technique. Automation of Electric Power Systems, 35(4), 65-69.

[9] Zimmerman, L., Lounsbury, D. W., \& Rosen, C. S. (2016). Participatory System Dynamics Modeling: Increasing Stakeholder Engagement and Precision to Improve Implementation Planning in Systems. Administration and Policy in Mental Health and Mental Health Services Research, 43(6), 834-849.

[10] Soares, da. Silva., Jéssica, P., Soares, B. G., \& Livi, S. (2017). Phosphonium-based ionic liquid as dispersing agent for MWCNT in melt-mixing polystyrene blends: Rheology, electrical properties and EMI shielding effectiveness. Materials Chemistry and Physics, 189, 162-168.

[11] [Kim, A. R., Park, J., \& Kim, Y. (2017). Quantification of performance shaping factors (PSFs)' weightings for human reliability analysis (HRA) of low power and shutdown (LPSD) operations. Annals of Nuclear Energy, 101, 375382. 